\title{
Are we ignoring the considerations for the dark-side of nanotechnology?
}

Volume 3 Issue 2 - 2016

\begin{abstract}
Editorial
With the inputs from all the branches of science into this magnificent field of Nanotechnology, it was not difficult for Nanotechnology buffs to dream up and fantasize many achievements. Due to virtually unlimited potential many fantasies did become true, this led to escalated excitement and today there are already more than 400 companies around the globe producing nano-transistors, nanodiodes, quantum computers, plasma displays and OLED, energy storing and producing products such as batteries, fuel cells, and solar cells, stronger and durable products for industries and automotives etc in large quantities.
\end{abstract}

Now use of Nanotechnology is being envisaged in the therapeutic and diagnostic field of medical and health care sciences also. Cancer therapeutics has generated interest in the scientists all over the world. But there will always be pros and cons to every growth and development achieved by human beings because nothing is ever perfect. Therefore, it is imperative to consider both advantages and disadvantages of Nanotechnology to determine the strategic direction of Nanoscience, We have to take lessons from earlier technologies. Past failures to consider environmental consequences have been costly and have consequences from semiconductor industry such as metals and solvents, synthetic chemicals like DDT and Freon, applications of natural compounds e.g. asbestos; and needless to say that development of transportation, air pollution, global warming, nuclear wastes, plastic wastes etc. Hence it is better to make early adjustments must introduce the environmental perspective early into the culture of emerging technologies (research and educational missions).

The question is, are we seriously looking into the considerations for the Dark-Side of Nanotechnology or are we ignoring it. Countries like United States, Japan, China, Korea, Germany and Britain are investing heavily in Nanotechnology research. But hardly 1\% of that goes into researching the risk assessment, despite the fact that nanotechnology also has the potential to cause harm to people and the environment. This is evident from the fact that thousands of papers are being published about the achievements in Nanoscience and Nanotechnology, but fewer of them have considered to examine how engineered nanoparticles will affect people and the environment.

However, some have seriously raised this consideration and there are some who are seriously working in assessing the dark side of Nanotechnology. Many governments and regulatory agencies related to environmental protection, FDA, occupational health and safety administrations are working on formulating regulations to guard against known as well as potentially harmful but unknown effects. Role of researchers become very difficult, because for each nanoparticles they have to find out whether the effect changes when materials take nano form and whether hazardousness is size and shape dependent. At present in many cases we do not have enough basic knowledge of the

\author{
Madhuri Sharon \\ Walchand Centre for Research in Nanotechnology and \\ Bionanotechnology, India \\ Correspondence: Madhuri Sharon, Walchand Centre for \\ Research in Nanotechnology and Bionanotechnology, Sholapur, \\ Maharashtra, India, Tel 918655723028 \\ Email shanmadhuri @gmail.com
}

Received: January 27, 2016 | Published: February I, 2016

properties and behaviour of the particles, what happens to the living cells when exposed to nanoparticles. There is a need to understand the nanoparticles and cell interaction.

It is heartening that scientists are paying attention to this concern. Centre for Responsible Nanotechnology is contributing to these issues." Department of Defence has given a grant of $\$ 5.5$ million to University of Rochester to study the effects of nanoparticles. Oxidative damage to brain of fish grown in presence of fullerene has been noted by Oberdorster she has speculated that the nanoparticles had stimulated the production of free radicals, highly reactive compounds that can cause cellular damage. Moreover, this result made it clear that nanoparticles can cross blood brain barrier and travel up to the nerve cells. Similarly, smaller particles of titanium dioxide nanoparticles that are used as pigments in white paint were found to cause more inflammation in rats and mice lung than an equal amount of larger particles when inhaled. Shevedova found that carbon nanotubes generated dangerous free radicals in cultures of human skin cells. Shape and size of $\mathrm{Cu}$ nanoparticles have been found to be lethal to zebra fish.

It is too early to tell whether and how different nano materials might harm people and the environment. But early studies show that this is something that should be looked into more seriously. Robert A. Freitas Jr., research scientist and author of Nanomedicine has said that

"We need to take seriously the prospects for molecular nanotechnology, personal nanofactories and nanorobotics, and attempt to understand the societal and political consequences of these technologies now, before it is too late. CRN is the only organization currently fulfilling this role."

Nanomedicine is relatively new field and it is mostly at experimental stage. Since, particles at nano-scale has very large surface to volume ratio (SVR) and other unique properties; it reacts in totally different way in comparison to their bulk material with living organisms. Thus, challenging our understanding about potential risks of nanoparticles. Use of photoactive nanomaterials and its use in the biological and biomedical fields needs exhaustive consideration and research. 
One of the great concerns is use of nanotechnology in food packaging industry for extending the freshness, detecting spoilage and inhibiting microbial growth. Question is whether nanoparticles such as silver that can kill microbes in food can also negatively affect human cells. There are both potential benefits and risks respiratory and digestive track (which is less worked out). Nanoparticles can travel to places in the body where other compounds cannot go, some nanoparticles can even move across the blood-brain barrier, or find their way across a placenta and into a foetus. Nanomaterials used in food, can find their way into the ambient environment through wastes from manufacturing, human waste disposal, and disposal of other wastes.

Similarly use of Nanotechnology for the environment for pollution prevention, may be cause of concern due to the materials used for production by-products and what happens when nano[article gets into the air, soil, water, or biota? No doubt Nanoscience, engineering, and technology is demand of the day but the health and environmental implications should be considered because most nanoparticles are not biodegradable.

\section{Conclusion}

In concluding paragraph I would like to say that apart from being concerned about devising safeguards against the possible risks of an accident, we have to also take a fresh look at the possibilities of a deliberate abuse of the technology. There is a need to establish a Nanotechnology Safety System that should encompass toxicological research, hazard identification and risk and exposure assessment as well as risk management.

\section{Acknowledgments}

None.

\section{Conflicts of interest}

None. 